\title{
Energy level alignment of electrically doped hole transport layers with transparent and conductive indium tin oxide and polymer anodes
}

\author{
Karsten Fehse, ${ }^{\text {a) }}$ Selina Olthof, Karsten Walzer, and Karl Leo \\ Institut für Angewandte Photophysik, ${ }^{\text {b) }}$ Technische Universität Dresden, D-01062 Dresden, Germany \\ Robert L. Johnson \\ Institut für Experimentalphysik, Universität Hamburg, Luruper Chaussee 149, D-22761 Hamburg, Germany \\ Hendrik Glowatzki, Benjamin Bröker, and Norbert Koch \\ Institut für Physik, Humboldt-Universität zu Berlin, Newtonstrasse 15, D-12489 Berlin, Germany
}

(Received 7 June 2007; accepted 6 August 2007; published online 15 October 2007)

\begin{abstract}
Using ultraviolet photoemission spectroscopy, we investigated the energy level alignment at the interfaces of typical anodes used in organic electronics, indium tin oxide (ITO) and poly(3,4-ethylenedioxythiophene):poly(styrenesulfonate) (PEDOT:PSS), with the oligomeric hole transport material $N, N, N^{\prime}, N^{\prime}$-tetrakis(4-methoxyphenyl)-benzidine (MeO-TPD), and studied the influence of electrical interface doping by the strong electron acceptor tetrafluoro tetracyanoquinodimethane $\left(\mathrm{F}_{4}-\mathrm{TCNQ}\right)$. The fundamentally different anode materials with work functions of $4.40 \mathrm{eV}$ (ITO) and $4.85 \mathrm{eV}$ (PEDOT:PSS) show different hole injection barriers, which also depend on the thickness of the $\mathrm{F}_{4}$-TCNQ interface dopant layer. PEDOT:PSS anodes exhibit a consistently lower hole injection barrier to MeO-TPD compared to ITO by $0.1 \mathrm{eV}$. We attribute this low hole injection barrier to additional charge transfer reactions at the PEDOT:PSS/MeO-TPD interface. In contrast, the deposition of the electron acceptor at the interface helps significantly to lower the hole injection barrier for ITO anodes. () 2007 American Institute of Physics.
\end{abstract}

[DOI: $10.1063 / 1.2786573$ ]

\section{INTRODUCTION}

The injection of charge carriers from electrodes into organic layers is an extremely important process in organic devices, such as organic light emitting diodes (OLEDs). So far, indium tin oxide (ITO) is the commonly used anode to inject holes into organic layers. However, often a large hole injection barrier (HIB) is found at the ITO/organic interface because the work function of ITO is in the range of 4.1-4.7 eV (depending on its pretreatment), which is significantly lower than the typical ionization energy of organic materials. ${ }^{1}$ Electrically doped charge transport layers may be used to enhance the injection of charge carriers from an ITO anode into organic layers, e.g., for OLEDs to achieve lower operating voltage and to increase power efficiency of the devices. $^{2}$ In such OLEDs, an electrically intrinsic organic emission layer is sandwiched in between two electrically doped organic layers for the $p$ and $n$ side, resulting in a so-called pin-OLED structure. Very high power efficiencies and long lifetimes of pin-OLEDs have been demonstrated using $\quad N, N, N^{\prime}, N^{\prime}$-tetrakis(4-methoxyphenyl)-benzidine (MeO-TPD) doped with tetrafluoro-tetracyanoquino dimethane $\left(\mathrm{F}_{4}-\mathrm{TCNQ}\right)$ as hole transport layer. ${ }^{3-5}$ The introduction of a strong electron acceptor such as $\mathrm{F}_{4}$-TCNQ in the hole transport layer serves several purposes simultaneously: (i) charge transfer between the anode material and $\mathrm{F}_{4}$-TCNQ lowers the hole injection barrier, ${ }^{6}$ (ii) charge transfer between the acceptor and the hole transport material acting as donor

\footnotetext{
${ }^{a}$ Electronic mail: karl.leo@iapp.de

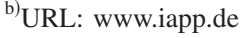

(e.g., MeO-TPD) modifies the density of states in the organic layer leading to $p$-type doping, ${ }^{7-9}$ and (iii) charge transfer increases the conductivity of the organic donor material compared to the pristine one. ${ }^{10}$ Another approach for efficient injection of holes into organic layers is the use of polymer layers based on poly(3,4-ethylenedioxythiophene): poly(styrenesulfonate) (PEDOT:PSS) with an ITO layer underneath. ${ }^{11,12}$ Commonly, the ITO layer is needed to provide a homogeneous lateral current distribution due to the several orders of magnitude lower conductivity of the polymer compared to metals or ITO. However, due to the very recent development of highly conductive formulations of PEDOT:PSS with low absorption in the visible, it is now possible to use PEDOT:PSS with a conductivity of up to $500 \mathrm{~S} / \mathrm{cm}$ as anode without the need of an inorganic layer underneath. ${ }^{13}$ In previous experiments, we found that hole injection from such a polymeric anode into a $p$-doped $\mathrm{MeO}-$ TPD layer was even superior to that from an ITO anode. ${ }^{13}$ This dependence of the hole injection efficiency on the nature of the anode material cannot be readily understood because the Fermi level in the $p$-doped MeO-TPD layer would be expected to be pinned, independent of the substrate. In order to clarify this issue, we performed ultraviolet photoelectron spectroscopy (UPS) experiments to directly assess the magnitude of HIBs for MeO-TPD layers on the inorganic and the polymeric conducting substrates in the presence of $\mathrm{F}_{4}$-TCNQ.

\section{EXPERIMENT}

UPS experiments were done at the FLIPPER II beam line at HASYLAB (Hamburg, Germany). ${ }^{14}$ ITO (120 nm on 
<smiles>CC(C)CC(CC(CC(CC(CC(C)C)c1ccc(S(=O)(=O)O)cc1)c1ccc(S(=O)(=O)O)cc1)c1ccccc1)c1ccccc1</smiles>

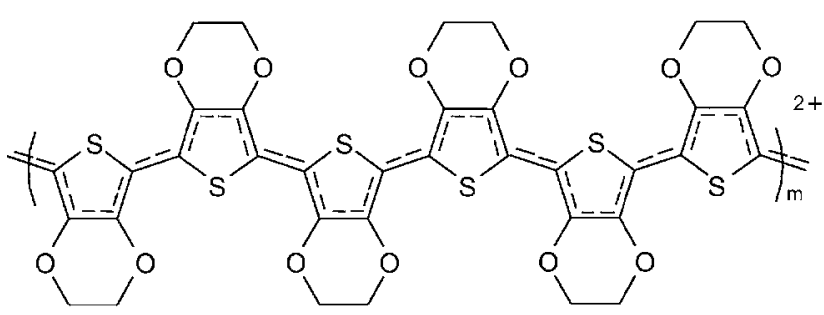

(b)

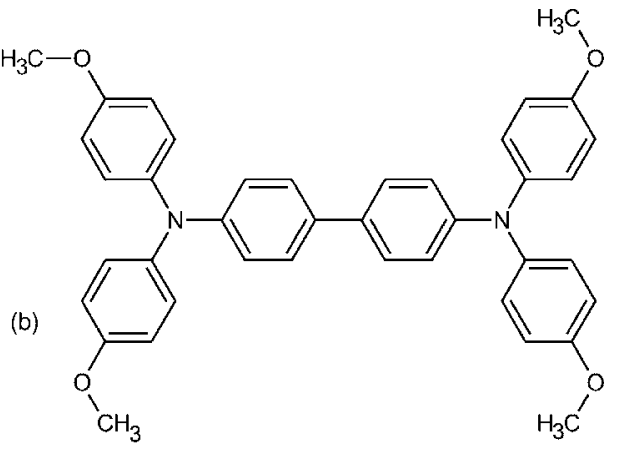

(c)

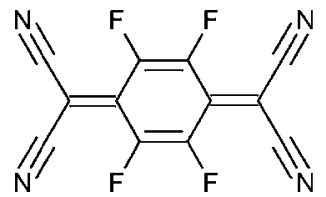

FIG. 1. Chemical structures of PEDOT:PSS (a), MeO-TPD (b), and $\mathrm{F}_{4}$-TCNQ (c).

glass, $25 \Omega / \square$ ) substrates were cleaned (sonication in deionized water, acetone, and methanol) and used for organic deposition without any further surface modification. A $70 \mathrm{~nm}$ thick film of PEDOT:PSS (Baytron PH 500 with 5 wt \% dimethylsulfoxide (DMSO), H.C. Starck) was spin coated onto these ITO substrates and subsequently heated in air for $20 \mathrm{~min}$ at $130{ }^{\circ} \mathrm{C}$ for drying. After an additional $10 \mathrm{~min}$ without heating, the substrates were inserted into ultrahigh vacuum (UHV). $\mathrm{F}_{4}$-TCNQ (one time sublimed, TCI Europe) and MeO-TPD (two times sublimed, Sensient) were evaporated from resistively heated pinhole sources in separate chambers in order to avoid cross contamination. The chemical structures of all materials used are shown in Fig. 1. The pressure in the analysis chamber was $3 \times 10^{-10}$ mbar. Photoelectron spectra were recorded with a double-pass cylindrical mirror analyzer at a photon energy of $22 \mathrm{eV}$. The secondary electron cut off (SECO) was measured at a sample bias of $-6 \mathrm{~V}$ and calculated from the intersection of the energy axis with the slope of the spectra. From these data, the interface dipole (ID) was calculated as the difference between the SECO of the oligomer film and the SECO of the bare substrate, which corresponds to the work function change of the substrate due to oligomer adsorption. We calculated the HIB from the difference between the Fermi level of the system and the leading edge of the peak derived from the highest occupied molecular orbital (HOMO) of the organic layers. All values have a numerical error of $\pm 0.05 \mathrm{eV}$, whereas the discussed energies can differ by $\pm 0.1 \mathrm{eV}$ between the samples.

\section{RESULTS AND DISCUSSION}

In order to discriminate between the effects of $\mathrm{MeO}$ TPD $p$-type doping (by $\mathrm{F}_{4}$-TCNQ) and HIB lowering due to charge transfer between the substrate and $\mathrm{F}_{4}$-TCNQ, the precoverage of ITO and PEDOT:PSS substrates with $\mathrm{F}_{4}$-TCNQ was varied as follows: $0.2 \AA / 0.5 \AA / 1 \AA$ and $5 \AA$. This systematic variation allows ruling out HIB lowering effects due to incomplete substrate coverage with the electron acceptor. ${ }^{6}$
Subsequent to depositing these thin acceptor layers, first $10 \AA$ and then an additional $20 \AA$ of MeO-TPD were evaporated, and UPS spectra were taken after every growth step. The work function $(\phi)$ values of individual pristine ITO and PEDOT:PSS substrates showed slight differences due to variations of water content and ubiquitous surface carbon contamination $^{15-18}$ because of exposure to air prior to loading into the UHV system. The average $\phi$ values were $4.40 \pm 0.10 \mathrm{eV}$ for ITO and $4.85 \pm 0.10 \mathrm{eV}$ for PEDOT:PSS.

The spectra of an ITO substrate before and after coverage with $0.5 \AA \mathrm{F}_{4}$-TCNQ and subsequently MeO-TPD are shown in Fig. 2. Since the spectra show only a minor dependence on different acceptor precoverages, we will discuss this sample in detail in the following. After deposition of $\mathrm{F}_{4}$-TCNQ, the SECO shifts to higher kinetic energy [Fig. $2(\mathrm{a})]$, corresponding to a $\phi$ increase (or ID) of $0.4 \mathrm{eV}$. This $\phi$ increase is indicative of a transfer of negative charge from ITO towards the electron acceptor $\mathrm{F}_{4}$-TCNQ, reminiscent of $\mathrm{F}_{4}$-TCNQ on Au. ${ }^{6}$ Interestingly, we observe only little changes in the valence region between clean ITO and that covered with the acceptor molecules [Fig. 2(c)]. Very similar observations are made for $\mathrm{F}_{4}$-TCNQ coverages up to $5 \AA$ (not shown). From this lack of clear $\mathrm{F}_{4}$-TCNQ related photoemission features, we conclude that there is a large portion of uncovered ITO surface, resulting in an extremely low UPS signal of the $\mathrm{F}_{4}$-TCNQ HOMO. All these observations can be rationalized when considering the presence of surfaceadsorbed carbon on air-exposed ITO already prior to $\mathrm{F}_{4}$-TCNQ deposition: Only bare ITO surface patches are available for the charge transfer with $\mathrm{F}_{4}$-TCNQ, whereas carbon covered areas are passivated. Once all available clean ITO patches are covered with $\mathrm{F}_{4}$-TCNQ, no further change of the sample work function is expected because surplus $\mathrm{F}_{4}$-TCNQ adsorbs in its neutral form, probably involving pronounced island growth. ${ }^{6}$ After deposition of $10 \AA \mathrm{MeO}-$ TPD, the photoemission peak derived from the oligomer $\mathrm{HOMO}$ is clearly visible (centered at $1.2 \mathrm{eV}$ binding energy) and a HIB of $0.35 \mathrm{eV}$ is measured. This value increases to $0.45 \mathrm{eV}$ after evaporation of an additional $20 \AA \mathrm{MeO}-\mathrm{TPD}$ 


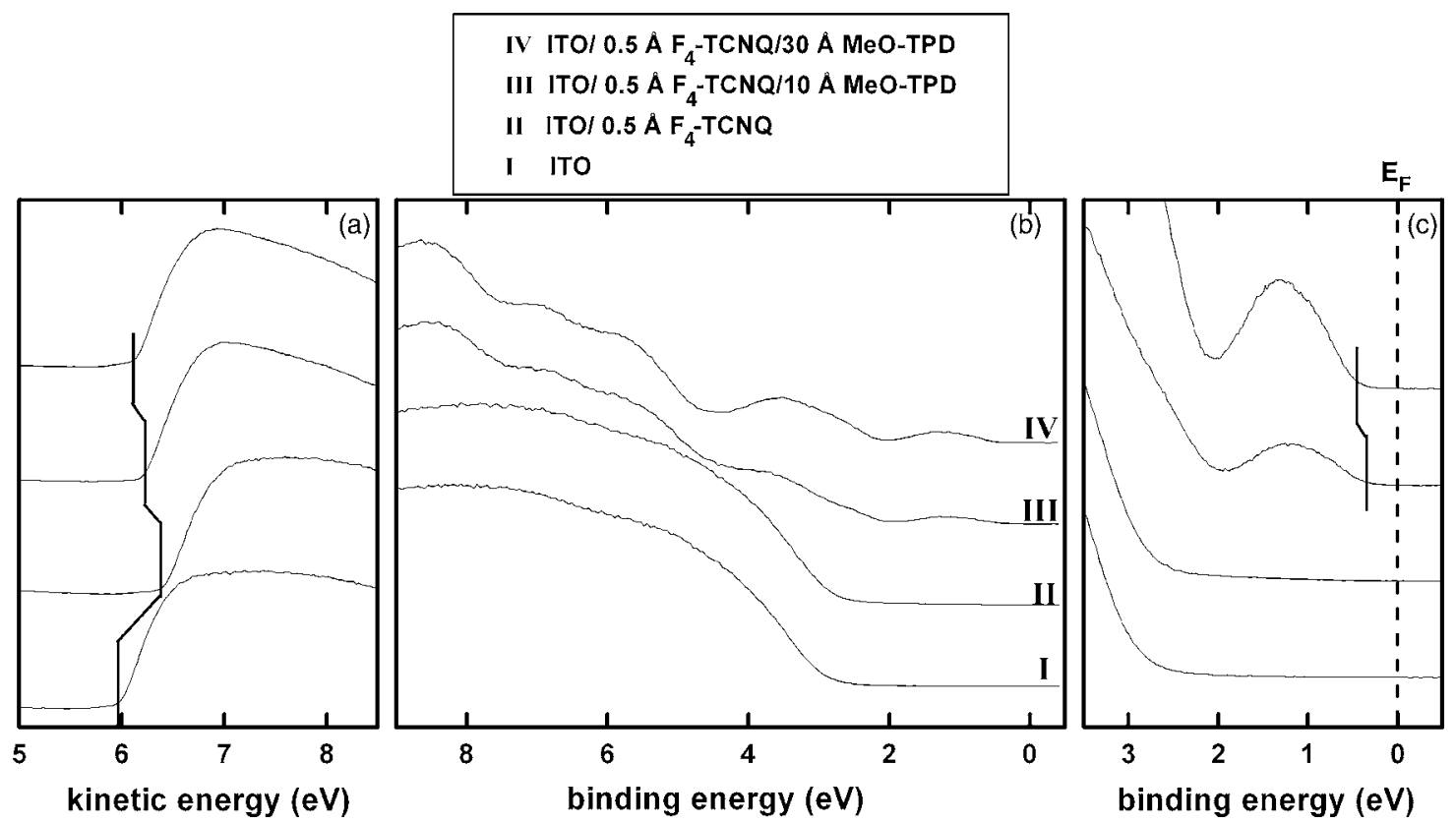

FIG. 2. UPS spectra of (I) a bare ITO substrate (II) with a coverage of $0.5 \AA \mathrm{F}_{4}$-TCNQ, (III) followed by $10 \AA$ and (IV) an additional $20 \AA$ MeO-TPD: (a) secondary electron cut off, (b) full spectrum, and (c) valence region.

due to a rigid shift of the entire spectrum toward higher binding energy. The SECO of the $10 \AA \mathrm{MeO}$-TPD film shifts relative to $0.5 \AA \mathrm{F}_{4}$-TCNQ/ITO by $-0.20 \mathrm{eV}$, corresponding to an ID of that magnitude. An additional $-0.1 \mathrm{eV}$ shift of the SECO is obtained for $30 \AA \mathrm{MeO}-\mathrm{TPD}$ coverage [Fig. 2(a)], which parallels the shift observed for the valence region. This could be a consequence of a lowering of the $\mathrm{MeO}$ TPD work function toward bulk material properties.

We will now discuss the properties of interfaces formed between a PEDOT:PSS substrate with the same $\mathrm{F}_{4}$-TCNQ and MeO-TPD coverages, namely, PEDOT:PSS/0.5 $\mathrm{F}_{4}$-TCNQ/10 $\AA \mathrm{MeO}-\mathrm{TPD}$ and an additional $20 \AA \mathrm{MeO}$ -
TPD (see Fig. 3). After deposition of $0.5 \AA \mathrm{F}_{4}$-TCNQ, we observe an ID of $0.10 \mathrm{eV}$ relative to the uncovered PEDOT: PSS, which is four times lower than for $0.5 \AA$ $\mathrm{F}_{4}$-TCNQ/ITO. This small increase of the sample work function upon acceptor coverage for PEDOT:PSS as compared to ITO is caused by the different nature of the conductive polymer layer as compared to ITO. PEDOT:PSS is an already doped polymer, since $\mathrm{PEDOT}^{+}$and $\mathrm{PSS}^{-}$moieties are present in the whole layer. Due to the admixture of a solvent (5 wt \% DMSO) to the PEDOT:PSS solution, the ratio of PEDOT and PSS at the surface of the film increases, but still an excess of insulating PSS is present at the polymer

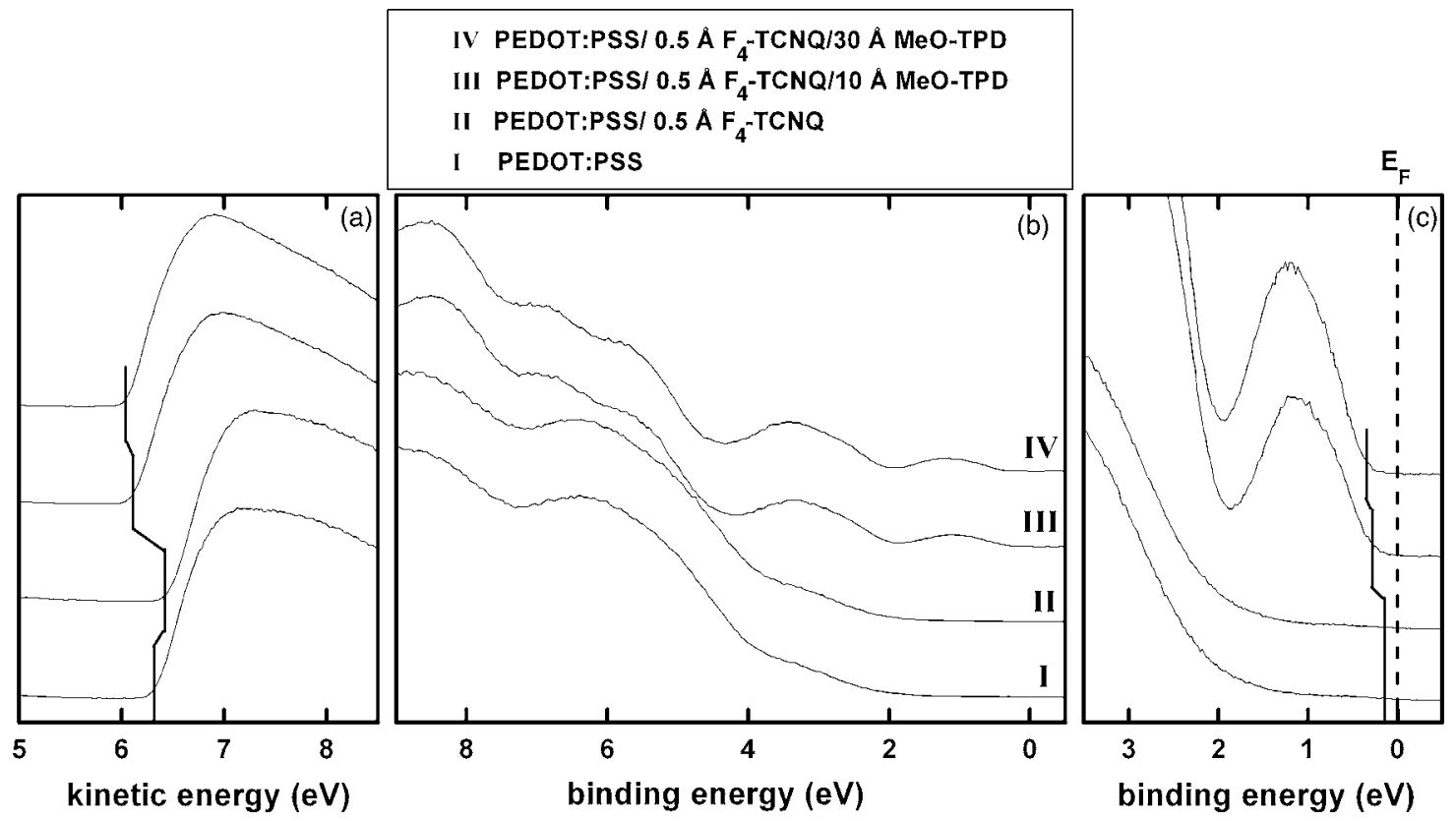

FIG. 3. UPS spectra of (I) a bare a PEDOT:PSS substrate (II) with a coverage of $0.5 \AA \mathrm{F}_{4}$-TCNQ, (III) followed by $10 \AA$ and (IV) an additional $20 \AA$ MeO-TPD: (a) secondary electron cut off, (b) full spectrum, and (c) valence region. 


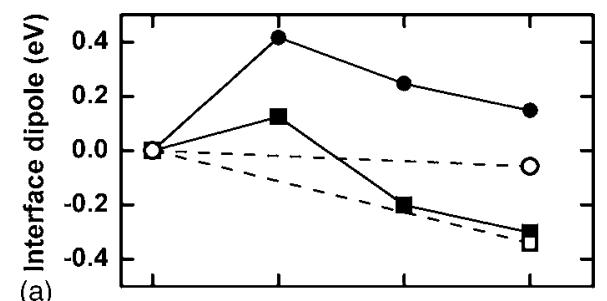

(a)

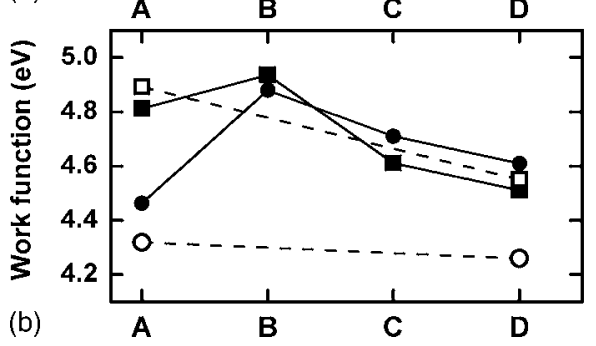

(A) $\rightarrow-$ PEDOT:PSS or $\rightarrow-$ ITO covered with

(B) 0.5 A F4-TCNQ

(C) $0.5 \AA$ A F4-TCNQ / $10 \AA \AA$ MeO-TPD

(D) $0.5 \AA$ F4-TCNQ/ $30 \AA$ MeO-TPD

(A) - $\square-$ PEDOT:PSS or - O- ITO covered with (D) only 30 Å MeO-TPD surface. ${ }^{19-21}$ Thus, if we assume a highly doped PEDOT:PSS layer (weight ratio of 1:2.5 of PEDOT to PSS) with an excess of PSS at the polymer surface, the probability of charge transfer from PEDOT to $\mathrm{F}_{4}$-TCNQ will be very low compared to ITO (as PEDOT is already oxidized) due to the significantly lower amount of ionizable surface. Charge transfer between $\mathrm{F}_{4}$-TCNQ and PSS can be ruled out on general grounds. After deposition of $10 \AA \mathrm{MeO}-\mathrm{TPD}$ onto the $\mathrm{F}_{4}$-TCNQ precovered PEDOT:PSS surface, the HIB for MeO-TPD is $0.25 \mathrm{eV}$ and the SECO shifts to lower kinetic energy by $-0.30 \mathrm{eV}$ (=ID). For $30 \AA \mathrm{MeO}-\mathrm{TPD}$ coverage, the HIB increases to $0.35 \mathrm{eV}$ [Fig. 3(c)], which is accompanied by a further SECO shift of $-0.10 \mathrm{eV}$ (as was the case for the ITO substrate in Fig. 2). This means that the HIB for p-doped MeO-TPD is $0.10 \mathrm{eV}$ smaller on PEDOT:PSS compared to ITO, which nicely explains the higher hole current measured for PEDOT:PSS substrates reported earlier. ${ }^{13}$

From the observations discussed above, we expect that the charge exchange reactions

$$
\begin{aligned}
\mathrm{PEDOT}^{+}+\mathrm{PSS}^{-}+\mathrm{MeO}-\mathrm{TPD}^{0} \rightarrow \mathrm{PEDOT}^{0}+\mathrm{PSS}^{-} & \\
& +\mathrm{MeO}-\mathrm{TPD}^{+}, \\
\mathrm{MeO}-\mathrm{TPD}^{0}+\mathrm{F}_{4}-\mathrm{TCNQ}^{0} \rightarrow & \mathrm{MeO}-\mathrm{TPD}^{+}+\mathrm{F}_{4} \\
& -\mathrm{TCNQ}^{-}
\end{aligned}
$$

occur at the conducting polymer/oligomer interface, which is facilitated by the low ionization energy of MeO-TPD $(\sim 4.9 \mathrm{eV}){ }^{22}$ Similar to experimental findings of pentacene and copper phthalocyanine at the PEDOT:PSS surface, PEDOT cations can "dope" the subsequently deposited MeO-TPD via charge transfer [Eq. (1)]. ${ }^{23,24}$ In addition, the doping of MeO-TPD by $\mathrm{F}_{4}$-TCNQ is a highly efficient process which also leads to a lower HIB [Eq. (2)]. ${ }^{7}$ Figure 4 summarizes the above results for the hole injection barriers interface dipoles, and work functions depending on the substrate and the sample composition. In order to investigate the impact of the thin acceptor precoverage on the interface properties, MeO-TPD was evaporated onto bare ITO and PEDOT:PSS substrates as well. The HIB for $30 \AA \mathrm{MeO}-$ TPD on bare ITO and PEDOT:PSS was determined to be 0.70 and $0.40 \mathrm{eV}$, respectively. Thus, the use of $\mathrm{F}_{4}$-TCNQ as precoverage is essential for ITO substrates to lower the HIB. In contrast, for PEDOT:PSS substrates, the HIB for MeOTPD without precoverage of $\mathrm{F}_{4}$-TCNQ is already lower than the one of MeO-TPD on precovered ITO. This is explained by the charge exchange reaction [Eq. (1)] occurring at the PEDOT:PSS/MeO-TPD interface.

The HIBs and IDs for different $\mathrm{F}_{4}$-TCNQ precoverages on both substrates obtained in further experiments are summarized in Fig. 5. Compared to ITO, the HIB is lower for all $\mathrm{F}_{4}$-TCNQ precoverages when a PEDOT:PSS substrate is used. The ID remains nearly constant for the PEDOT:PSS substrates and decreases for thicker $\mathrm{F}_{4}$-TCNQ layers on ITO substrates. The precoverage of ITO with $\mathrm{F}_{4}$-TCNQ causes a positive ID, whereas without precoverage, a nearly constant vacuum level is found. Most notably, there is no significant influence of the $\mathrm{F}_{4}$-TCNQ precoverage on the HIB for PEDOT:PSS. This may indicate that the reactions according to Eqs. (1) and (2) result in rather similar MeO-TPD cationic species. However, as the HIB is yet smaller for PEDOT:PSS substrates, we propose a somewhat different molecular conformation for $\mathrm{MeO}-\mathrm{TPD}^{+}$when being charge balanced by $\mathrm{PSS}^{-}$. Nevertheless, by using PEDOT:PSS for efficient hole

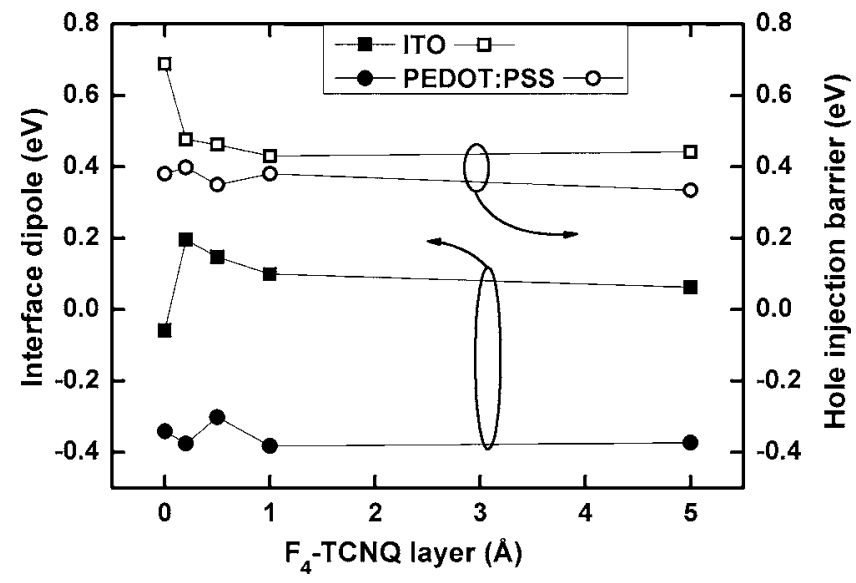

FIG. 5. Interface dipole and hole injection barrier on ITO or PEDOT:PSS substrates with different coverages of $\mathrm{F}_{4}$-TCNQ in between the anode and a $30 \AA$ thick MeO-TPD top layer. As marked by the arrows, open symbols represent the hole injection barrier (right axis), while solid symbols show the interface dipole (left axis). 
injection into the adjacent oligomer layers, the electrically doped charge transport layers and their high conductivity are beneficial, e.g., for the optimization of the layer thicknesses for maximum light extraction from the OLED to air. Additionally, lifetime experiments show that using doped transport layers is mandatory to decrease the degradation rate, which will be discussed elsewhere. Furthermore, doped transport layers can be used for efficient electron injection as well, a field where not much progress was made with polymers in the last few years.

\section{CONCLUSION}

Using ultraviolet photoemission spectroscopy, we analyzed the electronic properties of MeO-TPD in contact with two typical anode materials used in organic electronics, ITO and PEDOT:PSS. For lowering the hole injection barrier, we used a thin interface layer made of the strong electron acceptor $\mathrm{F}_{4}$-TCNQ, a typical dopant for MeO-TPD. In the case of ITO anodes, a strong reduction of the hole injection barrier was found when a thin film of $\mathrm{F}_{4}$-TCNQ was deposited in between the anode and MeO-TPD. In contrast, we observed a low barrier for hole injection from PEDOT:PSS into MeOTPD, both with and without $\mathrm{F}_{4}$-TCNQ at the interface. In any case and independently of the thickness of the electron acceptor interlayer, the hole injection barrier from PEDOT: PSS into MeO-TPD is $0.1 \mathrm{eV}$ lower than for ITO. We attribute this lower energy barrier mainly to charge transfer reactions between the polymer and the oligomers.

\section{ACKNOWLEDGMENTS}

We thank Dr. M. Pfeiffer from Heliatek GmbH, as well as Dr. W. Lövenich and Dr. A. Elschner from H.C. Starck $\mathrm{GmbH}$ for helpful discussions and for providing PEDOT:PSS. This work was funded by the European Commission under Contract Nos. IST-2002-004607 (project acronym "OLLA") and NMP-3-CT-2006-033197 ("ICONTROL") and by the German BMBF under Contract No. 13 N8855, project acronym "Rollex". N.K. acknowledges financial support by the Emmy-Noether-Program (DFG).

${ }^{1}$ D. J. Milliron, I. G. Hill, C. Shen, A. Kahn, and J. Schwartz, J. Appl. Phys. 87, $572(2000)$

${ }^{2}$ K. Walzer, B. Maennig, M. Pfeiffer, and K. Leo, Chem. Rev. (Washington, D.C.) 107, 1233 (2007).

${ }^{3}$ G. He, M. Pfeiffer, K. Leo, M. Hofmann, J. Birnstock, R. Pudzich, and J. Salbeck, Appl. Phys. Lett. 85, 3911 (2004).

${ }^{4}$ G. Schwartz, K. Fehse, M. Pfeiffer, K. Walzer, and K. Leo, Appl. Phys. Lett. 89, 083509 (2006).

${ }^{5}$ R. Meerheim, K. Walzer, M. Pfeiffer, and K. Leo, Appl. Phys. Lett. 89, 061111 (2006).

${ }^{6}$ N. Koch, S. Duhm, J. P. Rabe, A. Vollmer, and R. L. Johnson, Phys. Rev. Lett. 95, 237601 (2005).

${ }^{7}$ J. Blochwitz, T. Fritz, M. Pfeiffer, K. Leo, D. M. Alloway, P. A. Lee, and N. R. Armstrong, Org. Electron. 2, 97 (2001).

${ }^{8}$ R. Schmechel, J. Appl. Phys. 93, 4653 (2003).

${ }^{9}$ O. Tal, Y. Rosenwaks, Y. Preezant, N. Tessler, C. K. Chan, and A. Kahn, Phys. Rev. Lett. 95, 256405 (2005).

${ }^{10}$ B. Maennig, M. Pfeiffer, N. Nollau, X. Zhou, K. Leo, and P. Simon, Phys. Rev. B 64, 195208 (2001).

${ }^{11}$ J. C. Scott, S. A. Carter, S. Karg, and M. Angelopoulos, Synth. Met. 85, 1197 (1997).

${ }^{12}$ Y. Zhang, F. He, G. Cheng, C. Ruan, Y. Lin, Y. Zhao, Y. Ma, and S. Liu, Semicond. Sci. Technol. 22, 214 (2007).

${ }^{13}$ K. Fehse, K. Walzer, K. Leo, W. Lövenich, and A. Elschner, Adv. Mater. (Weinheim, Ger.) 19, 441 (2007).

${ }^{14}$ R. L. Johnson and J. Reichardt, Nucl. Instrum. Methods Phys. Res. 208, 791 (1983).

${ }^{15}$ Separate x-ray photoelectron spectroscopy measurements of solventcleaned ITO revealed typically 30 at. \% carbon at the surface.

${ }^{16}$ N. Koch, A. Vollmer, and A. Elschner, Appl. Phys. Lett. 90, 043512 (2007).

${ }^{17}$ A. Wan, J. Hwang, F. Amy, and A. Kahn, Org. Electron. 6, 47 (2005).

${ }^{18}$ S. Rentenberger, A. Vollmer, E. Zojer, R. Schennach, and N. Koch, J. Appl. Phys. 100, 053701 (2006).

${ }^{19}$ X. Crispin, F. L. E. Jakobsson, A. Crispin, P. C. M. Grim, P. Andersson, A. Volodin, C. van Haesendonck, M. van der Auweraer, W. R. Salaneck, and M. Berggren, Chem. Mater. 18, 4354 (2006).

${ }^{20}$ J. Hwang, F. Amy, and A. Kahn, Org. Electron. 7, 387 (2006).

${ }^{21}$ G. Greczynski, T. Kugler, and W. R. Salaneck, Thin Solid Films 354, 129 (1999).

${ }^{22} \mathrm{At}$ a different UPS setup, we measure $5.0 \mathrm{eV}$.

${ }^{23}$ H. Peisert, M. Knupfer, F. Zhang, A. Petr, L. Dunsch, and J. Fink, Surf. Sci. 566, 554 (2004)

${ }^{24}$ N. Koch, A. Elschner, J. P. Rabe, and R. L. Johnson, Adv. Mater. (Weinheim, Ger.) 17, 330 (2005). 